\title{
The research of mobile sensor nodes scheduling optimization based on
}

\section{GA-AFSA}

\author{
Zhang Xiao-feng ${ }^{1, a}$, Yu Li-juan ${ }^{2, b}$, Mao Wen-wu ${ }^{1, c}$, \\ Chen Cheng-ming ${ }^{1, d}$, Xia Jun ${ }^{2, e}$ \\ ${ }^{1}$ College of Engineering, Shanghai Ocean University, Shanghai ,China; \\ ${ }^{2}$ College of Mechanical and Energy Engineering, Tongji University, Shanghai, China \\ a1020744311@qq.com, bljyu@shou.edu.cn, ${ }^{\mathrm{c}} 1391212298 @ q q . c o m$, \\ d252765682@qq.com, ${ }^{\mathrm{e}} 55312658 @ q q . c o m$
}

Key words: WSN; Genetic algorithm; Artificial fish algorithm; sensor nodes Scheduling

Abstract: in order to make up for sensor nodes' death effectively, this paper studies a kind of artificial fish algorithm (AFSA) improved by genetic algorithm (GA) to optimize the project of mobile sensor nodes Scheduling and the coverage of Wireless Sensor Networks (WSN) and energy consumption will be as the optimization goal. This paper introduce crossover and mutation probability of GA to improve AFSA. The simulation experiments have proved that The global search ability of GA-AFSA is better, and the improved GA-AFSA has higher efficiency and quality of the algorithm.

\section{Introduction}

Wireless Sensor Network (WSN) is a kind of wireless communication network which is composed of various sensors, which transmits the data to the network coordinator through the multi hop self-organizing mode. Among them, the deployment scheme of sensor nodes is the primary problem of the whole sensor network, it is directly related to the communication effect of each node and the comprehensiveness of data acquisition. Usually, sensor nodes have limited energy, and the energy consumption of the sensor nodes can easily lead to the problem of node death, and the death sensor nodes directly affect the communication effect WSN. About the mobile sensor node scheduling problem, domestic and foreign research institutions have carried out the corresponding research. Jia et al. proposed a coverage strategy based on Genetic Algorithm in the wireless sensor network, but the genetic algorithm has a slow convergence speed. Zhou et al. proposed a hybrid network node deployment method based on virtual force, but there is still a large space for the sensor coverage to improve. Zhou et al proposed the coverage optimization strategy of wireless sensor networks based on AFSA, but AFSA has the problem of early convergence and is easy to fall into local optimum. Lan et al proposed a wireless sensor network coverage optimization strategy based on improved AFSA, but its energy balance model is not suitable for mobile nodes.

At present, most of the research is based on situation that all sensor nodes are static, so it's not meet the application of some network environment. In this paper, the principle of maximum coverage and energy consumption will be considered, and the model analysis is carried out to make the mobile sensor scheduling problem better. This paper use crossover and mutation probability of 
GA to improve the global search ability of ASFA and solve the problem of early convergence in ASFA.

\section{The Scheduling optimization model analysis of mobile sensors}

\section{Problem description and analysis}

there is a shape of a rectangle to be monitored with area of $\mathrm{S}, \mathrm{Z}$ monitoring sensor (including $\mathrm{M}$ mobile sensor nodes and $\mathrm{N}$ fixed sensor nodes )is putted on the monitoring ground .when fixed sensor nodes died, the mobile sensor will be dispatched to make up for the decrease in the coverage, at the same time, in order to extend the service life of WSN ,the sensor node energy consumption will be balanced. In this paper, the rectangle to be detected area is abstracted into $\mathrm{P}^{*} \mathrm{Q}$ pixels, and the coverage model and energy consumption model are established.

\section{Coverage model}

In real life, with the increasing of the distance between the monitoring points and the sensor, the sensing quality is also lost. The success rate that pixel $\mathrm{S}$ can be monitored by sensor $\mathrm{C}$ is expressed as $\mathrm{P}(\mathrm{C}, \mathrm{S})$, and the distance between $\mathrm{S}$ and $\mathrm{C}$ is shorter, the success rate will be higher. As shown in Eq. 1:

$$
P(c, s)=\left\{\begin{array}{c}
1, \operatorname{dis}(c, s) \leq r a \\
e^{-\lambda(\operatorname{dis}(c, s)-r a)^{k}}, r a<\operatorname{dis}(c, s) \leq R \\
0, \operatorname{dis}(c, s)>R
\end{array}\right.
$$

Among them, $\mathrm{k}$ and $\lambda$ are the sensor adjustable parameters, which reflects different physical characteristics between different sensors; dis (c, s) is the distance between c and s; ra is threshold; $\mathrm{R}$ is the sensing radius. Due to the presence of $\mathrm{P}(\mathrm{C}, \mathrm{S})$, sometimes in order to make the monitoring data more reliable, the pixel will be monitored jointly, thus, the coverage of all pixels to be shown in Eq. 2:

$$
f 1=\frac{\sum_{j=1}^{P^{*} Q}\left\{1-\prod_{i=1}^{Z}\left[1-P\left(c_{i}, s_{j}\right)\right]\right\}}{P * Q}
$$

\section{Energy balance model}

In order to compensate for the death of the sensor nodes, the scholars have designed a mobile sensor, which has more energy than ordinary sensor nodes, but it also brings more energy consumption. Assuming that the energy consumption of the sensor is proportional to the moving distance, in order to balance the energy consumption of the mobile sensor nodes, the node with higher residual energy will choose the far route. As a result, the energy balance function is shown in Eq. 3:

$$
f 2=L^{*}\left[\sum_{i=1}^{M} \frac{K^{*} \operatorname{dis}\left(c_{i}, \text { clast }_{i}\right)^{*} E(C)}{E\left(c_{i}\right)}\right]^{-1}
$$

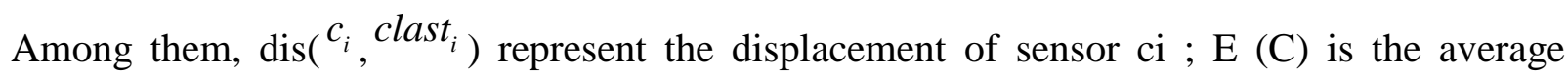
residual energy of all the mobile sensors; $\mathrm{E}(\mathrm{ci})$ is the residual energy of sensor ci ; $\mathrm{K}$ and $\mathrm{L}$ is the scale coefficient, take $\mathrm{K}=1, \mathrm{~L}=0.5^{*}(\mathrm{P}+\mathrm{Q})$. 


\section{Mobile node optimization function}

In order to make the mobile sensor nodes can give priority to the sensor coverage, the second is to make the energy consumption is lower and balanced. In this paper, the fitness function of the mobile node can be weighted by the coverage function and the energy consumption function, as shown in Eq. 4:

$$
Y=w 1 * f 1+w 2 * f 2
$$

$\mathrm{w} 1$ is the weight of coverage function, taking $\mathrm{w} 1=0.8$; w 2 is the weight of energy consumption function, taking $\mathrm{w} 2=0.2$.

\section{The mobile sensor node scheduling based on GA- AFSA}

\section{AFSA part in the GA-AFSA}

AFSA is a new type of artificial intelligence group optimization algorithm. By simulating the fish swimming to optimize solution of the function, its global search ability and robustness is good, which is not influenced by the initial state of the model. In AFSA, each artificial fish is a solution, and then through the interaction and cooperation to complete the problem solving. Aiming at solving the problem of sensor network mobile node scheduling problem, the solution space is described as a fish swarm with Fish_no artificial fish, then the scheduling target coordinate matrix of all mobile nodes $\left(\mathrm{C}_{1} ; \mathrm{C}_{2} ; \mathrm{C}_{3} ; \ldots ; \mathrm{C}_{\mathrm{M}}\right)$ abstract as an artificial fish $\mathrm{F}_{\mathrm{j}}$. the main behavior of each artificial fish:

1) foraging behavior:Artificial fish $F_{j}$ in the field of visual range choose a comparison fish $F_{k}$ randomly, that is, to meet the norm $\left\|F_{j}-F_{k}\right\|<$ Visual, through comparing the food concentration of the respective waters to determine whether to move a step in the direction of fish $F_{k}$, if the waters of $F_{k}$ have better food concentration, then move, otherwise try to choose again, if Try_no times the better concentration has not been found, walk in the field of visual range randomly. as shown in Eq. 5:

$$
F_{j}=F_{j}+\text { rand }^{*} \text { Step } * \frac{F_{k}-F_{j}}{\left\|F_{k}-F_{j}\right\|}
$$

2) the following behavior:After searching, fish $F_{j}$ found a total of near_no fish near it, one of these neighbors have has the largest food concentration, then fish $\mathrm{F}_{\mathrm{j}}$ compare with the Fs, if the Fs has better food concentration, $\mathrm{Fj}$ move a step in the direction of fish $\mathrm{F}_{\mathrm{S}}$, otherwise attempt to clustering behavior.

3) clustering behavior:The near_no fish is composed of a fish swarm $(\mathrm{Fa}, \mathrm{Fb}, \mathrm{Fc}, .$.$) , though$ comparing food concentration between the center position of the fish and $F_{j}$, if the center has better food concentration and the swarm do not hit the maximum congestion, $F_{j}$ move to the center of the fish in a step, otherwise again try foraging behavior.

\section{The improving part of GA-AFSA}

The basic AFSA use the bulletin board to record the artificial fish individual and its food concentration(the mobile sensor locations and the best fitness).After moving the fish will compare to the data in the bulletin board, if better replace it and update bulletin board. When the data of the bulletin board is not changed, it may be because some of the artificial fish is in the local optimal position, which affects the convergence speed and the global nature of the algorithm. Thus, the variation and crossover operator of GA are introduced to deal with the local optimum. When the frequency that sensor position matrix and fitness change in the bulletin board has not changed reach 
the maximum Best_no, the variation and crossover operator will be executed Gen_no times:

1)cross behavior: the behavior that gene from the two generation fish will be recombined to produce new generation, so as to improve the search range of genetic algorithm. when the crossover probability is great, the algorithm will have a strong search ability, but it is also easy to make a better artificial fish gene is destroyed; when the crossover probability is small, the convergence rate of the algorithm will slow down. This paper introduce a mutative crossover probability, so that the better fish gene will select the great crossover probability, the worse fish will select the small, as shown in Eq. 6:

$$
P_{c}=\left\{\begin{array}{c}
P 1-(P 1-P 2) \frac{\left(Y^{\prime}-Y_{a v g}\right)}{\left(Y_{\max }-Y_{a v g}\right)}, Y^{\prime} \geq Y_{a v g} \\
P 1 Y^{\prime} \leq Y_{a v g}
\end{array}\right.
$$

Among them, $\mathrm{Y}$ 'is the better fish's fitness; $\mathrm{Y}_{\text {avg }}$ is the average fitness of the fish outside the bulletin board; P1, P2 are the maximum crossover probability and minimum crossover probability, take $\mathrm{P} 1=0.8, \mathrm{P} 2=0.2$. After the cross behavior, the sub generation fish compares with the bulletin board, if it better than the bulletin board, then update the bulletin board.

2) mutation behavior: the variation behavior is what chooses a fish outside the bulletin board randomly and then change it randomly. The occurrence probability of mutation is very low, usually take a very small value, this paper takes that the mutation probability equal 0.2 . After mutation, the fish will compare with the bulletin board, if better than the bulletin board, then update the bulletin board.

\section{Calculation process of the GA-AFSA}

1) the initial setting of the AFSA parameters: Set iteration number is 0 , in the feasible region ,generate Fish_no fish swarm randomly.

2) bulletin board assignment: Calculate each fish's fitness function, which has the maximum value of the fish will assign to the bulletin board.

3) fish behavior: If the fish's fitness is lower than the average level, the fish will carry out the following behavior to increase their access to food; whereas the fish will carry out clustering behavior.

4) bulletin board update. The artificial fish will be compared with the finess function after every move. If the value is higher than that before moving, update bulletin board.

5) GA behavior. When the times that bulletin board's value was not changed hit maximum, cross behavior and mutation behavior will be carried out. GA behavior will continue GA_no times. Then go to step 5 .

6) termination judgment. When iterations to reach the maximum, then terminate the algorithm, otherwise go to step 3 .

7) algorithm output. The artificial fish in the bulletin board is the optimal scheduling scheme, which will be the output.

\section{Simulation Analysis}

Assuming the wireless sensor network monitoring area is $700 \mathrm{~m} * 800 \mathrm{~m}$, the sensing radius of each sensor node is $80 \mathrm{~m}$, and the sensor scheduling scheme is simulated by MATLAB 2012. The rectangle detection area contains $351 * 401$ pixels, and the dark circle is mobile sensor nodes with a total of 14, and the light circle is fixed sensor nodes with a total of 16 . 
Setting the sensors distribution as Fig1 and its coverage is $79.56 \%$. Setting the maximum of iterations is 200 , the number of fish swarm is 20 , the maximum congestion is 0.5 , the visual range is $35 \mathrm{~m}$, the artificial fish is 0.5 , the try_no is 6 , the maximum that board do not change is 10 ; $\mathrm{L}$ is 750 ; $\mathrm{K}=1$; the initial energy of each node is $1 \mathrm{~J}$.

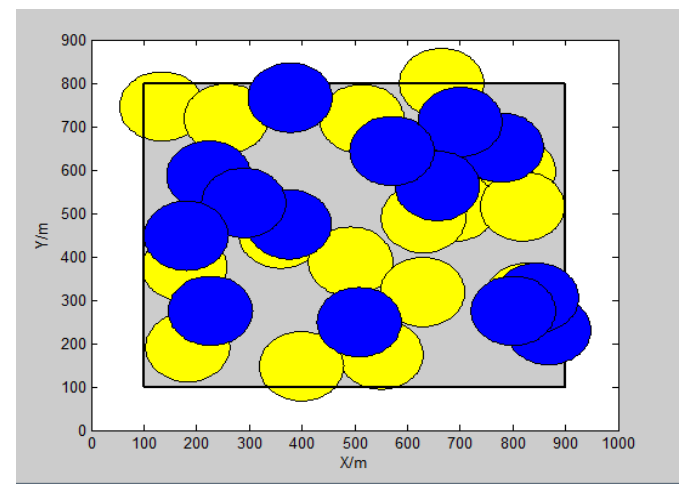

Fig.1 initial distribution

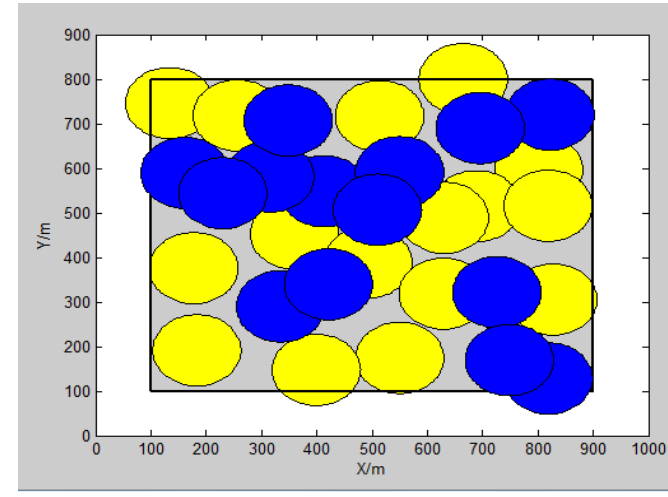

Fig.2 AFSA operation

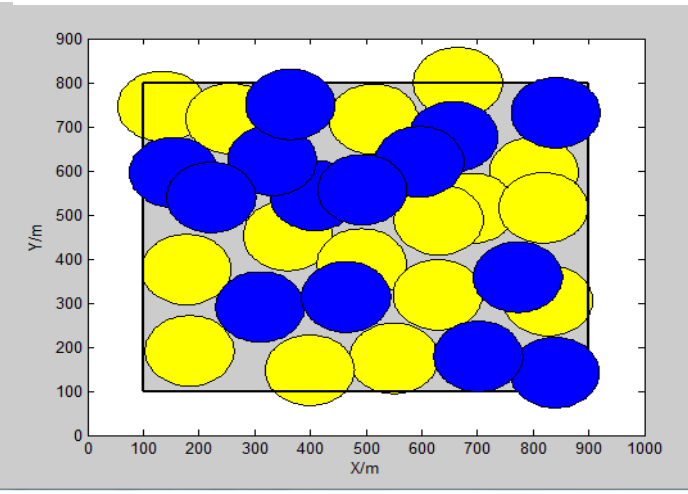

Fig.3 GA-AFSA operation

If the mobile sensors node Scheduling optimization based on AFSA, it's shown as Fig2,the sensor network coverage is $91.45 \%$.the value of energy consumption function is 0.569 . At the same time, Scheduling optimization based on GA-AFSA, it's shown as Fig3, the sensor network coverage is $94.63 \%$, the value of energy consumption function is 0.612 .From this, it can be seen that the use of GA-AFSA compare with AFSA, the coverage increase by $3.18 \%$, the energy consumption of the function value increase by 0.143 .

As shown in Table 1, with the increase of the number of iterations, the value of the fitness function of the mobile node gradually tends to converge, the basic artificial fish swarm algorithm (AFSA) compare with the improved artificial fish swarm algorithm(GA-AFSA), their solving efficiency is almost the same, but in the late stage, the AFSA has a premature convergence. Thus, the GA-AFSA can accomplish the optimization of mobile sensor node scheduling better.

Table 1 Comparison between AFSA and GA-AFSA

\begin{tabular}{lllll}
\hline & \multicolumn{4}{c}{ Iterations } \\
\cline { 2 - 5 } & 50 & 100 & 150 & 200 \\
\hline AFSA & 0.7722 & 0.8116 & 0.8377 & 0.8454 \\
\hline GA-AFSA & 0.7633 & 0.8389 & 0.8601 & 0.8794 \\
\hline
\end{tabular}




\section{Conclusions}

In this paper, the coverage model and energy consumption model are established and carry out GA-AFSA to improve the global search ability of AFSA, and also the convergence speed of the algorithm is improved. Through the simulation experiments, the improved artificial fish swarm algorithm can better solve the problem of mobile sensor node scheduling optimization, so that the sensor network coverage is better, the duration of network is longer, and the accuracy and reliability of the transmission data is ensured.

\section{Acknowledgements}

This work was financially supported by the Shanghai university knowledge service platform project (ZF1206)

\section{References}

[1] Jia Jie, Chen Jian, Chang Guiran, et al. Optimal coverage mechanism based on Genetic Algorithm in wireless sensor networks. Control and decision, 2007; 22 (11): 1289 - 1292

[2] Su Caihong, Lin Meijin, Wang Fei. Research on Optimization Algorithm of wireless sensor network coverage. Computer simulation, 2011; 28 (3): 178 - 181

[3] Zhou Tong, Bingrong Hong, Pu Songhao. Hybrid sensor networks deployment based on virtual force. The research and development of computer, 2007; 44 (6): 965 - 972

[5] Brant RE. A comment on'graph-based algorithm for Boolean function manipulation'[J]. IEEE Trans on Computers [5], 2000,49 (11): 1290-1292.

[6] Zhou Yongquan, Yao Xiangguang, Li Yongmei. Hybrid optimization algorithm of artificial fish swarm and particle swarm optimization (2010): 27 (6): 2084 - 2086. 